\title{
Subthalamic theta activity: a novel human subcortical biomarker for obsessive compulsive disorder
}

Pnina Rappel ${ }^{1,2}$, Odeya Marmor ${ }^{1,2}$, Atira S Bick ${ }^{1,3}$, David Arkadir ${ }^{3}$, Eduard Linetsky ${ }^{3}$, Anna Castrioto ${ }^{4}$, Idit Tamir ${ }^{3,5,6}$, Sara A. Freedman ${ }^{3,7}$, Tomer Mevorach ${ }^{3}$, Moran Gilad ${ }^{3}$, Hagai Bergman $\mathbb{1}^{1,2}$, Zvi Israel ${ }^{3,5}$ and Renana Eitan ${ }^{1,3,8}$

\begin{abstract}
Obsessive-compulsive disorder (OCD) is a common and serious psychiatric disorder. Although subthalamic nucleus deep brain stimulation (DBS) has been studied as a treatment for OCD patients the underlying mechanism of this treatment and the optimal method of stimulation are unknown. To study the neural basis of subthalamic nucleus DBS in OCD patients we used a novel, implantable DBS system with long-term local field potential sensing capability. We focus our analysis on two patients with OCD who experienced severe treatment-resistant symptoms and were implanted with subthalamic nucleus DBS systems. We studied them for a year at rest and during provocation of OCD symptoms (46 recording sessions) and compared them to four Parkinson's disease (PD) patients implanted with subthalamic nucleus DBS systems (69 recording sessions). We show that the dorsal (motor) area of the subthalamic nucleus in OCD patients displays a beta $(25-35 \mathrm{~Hz})$ oscillatory activity similar to PD patients whereas the ventral (limbic-cognitive) area of the subthalamic nucleus displays distinct theta $(6.5-8 \mathrm{~Hz})$ oscillatory activity only in OCD patients. The subthalamic nucleus theta oscillatory activity decreases with provocation of OCD symptoms and is inversely correlated with symptoms severity over time. We conclude that beta oscillations at the dorsal subthalamic nucleus in OCD patients challenge their pathophysiologic association with movement disorders. Furthermore, theta oscillations at the ventral subthalamic nucleus in OCD patients suggest a new physiological target for OCD therapy as well as a promising input signal for future emotional-cognitive closed-loop DBS.
\end{abstract}

\section{Introduction}

Obsessive compulsive disorder (OCD) is a debilitating psychiatric disorder characterized by obsessions and compulsions. Although understanding of the etiology of OCD is limited, functional and structural brain imaging studies have revealed aberrant cortico-striato-thalamocortical (CSTC) circuits in $\mathrm{OCD}^{1,2}$. The subthalamic nucleus (STN) has a central position within the CSTC

\footnotetext{
Correspondence: Renana Eitan (REITAN@partners.org)

'Department of Medical Neurobiology (Physiology), Institute of Medical Research - Israel-Canada, the Hebrew University-Hadassah Medical School, Jerusalem, Israel

${ }^{2}$ The Edmond and Lily Safra Center for Brain Research, the Hebrew University, Jerusalem, Israel

Full list of author information is available at the end of the article
}

These authors contributed equally: Pnina Rappel, Odeya Marmor.

(c) The Author(s) 2018 circuits and through its sensorimotor, emotional and cognitive areas coordinates decision-making, actionselection and various high-order processes ${ }^{3-6}$. STN deep brain stimulation (DBS) has been studied as a treatment for severe resistant OCD patients ${ }^{6-13}$. DBS surgery allows both acute and chronic electrophysiological recordings from DBS targets.

Synchronized oscillatory activity is a feature of healthy brain that facilitates communication between nearby and distant neuronal networks ${ }^{14}$. Pathological variations in oscillatory activity may represent brain disorders. In Parkinson's disease, excessive synchronized low $(12-25 \mathrm{~Hz})$ and high $(25-35 \mathrm{~Hz})$ beta band activity in the dorsal STN has been identified as pathognomonic and thought to be responsible for some of the parkinsonian

\footnotetext{
Open Access This article is licensed under a Creative Commons Attribution 4.0 International License, which permits use, sharing, adaptation, distribution and reproduction in any medium or format, as long as you give appropriate credit to the original author(s) and the source, provide a link to the Creative Commons license, and indicate if changes were made. The images or other third party material in this article are included in the article's Creative Commons license, unless indicated otherwise in a credit line to the material. If material is not included in the article's Creative Commons license and your intended use is not permitted by statutory regulation or exceeds the permitted use, you will need to obtain
} permission directly from the copyright holder. To view a copy of this license, visit http:/creativecommons.org/licenses/by/4.0/. 
symptoms such as rigidity and akinesia ${ }^{15-17}$. Therapeutic STN DBS reversibly reduces both this pathologic activity and parkinsonian motor symptoms ${ }^{18}$.

Although many studies have investigated the subregions of the STN in PD patients, only two groups have reported acute STN recordings in OCD patients ${ }^{19-}$ 23 . These invasive human recordings in a small group of patients have been recorded with intraoperative leads or temporarily externalized leads in the immediate postoperative period. Nonspecific oscillations have been reported in OCD but the relationship to obsessions and compulsions was not clear mainly because OCD symptoms change over time, have specific triggers related to location, timing or situation and are typically reduced immediately following the insertional effects of DBS surgery. To circumvent these difficulties, we used an investigational device, activa $\mathrm{PC}+\mathrm{S}$ (Medtronic Inc.), that in addition to standard stimulating capabilities, can also record long-term local field potentials (LFPs). This system allows long term functional electrophysiological recordings of the STN throughout different phases of the disease, behavioral challenges and DBS treatment.

\section{Methods}

\section{Study participants}

In this study, two OCD patients and a control group of four PD patients underwent STN DBS surgery with implantation of the Activa $\mathrm{PC}+\mathrm{S}$ pacemaker (Medtronic, Inc, Minneapolis, MN, USA). All patients met accepted inclusion criteria for DBS surgery and signed informed consent. PD patients had (i) advanced idiopathic PD; (ii) long-term levodopa use with reduced efficacy, on-off motor fluctuations and increased incidence of medication-induced side effects; (iii) normal cognitive function or mild-moderate cognitive decline as defined by Addenbrooke's cognitive examination (ACE) $>75$ and frontal assessment battery $(\mathrm{FAB})>10$. OCD patients were diagnosed by Structured Clinical Interview for DSM-IV (SCID-IV) and had (i) disabling severe symptoms, as assessed by a Yale-Brown Obsessive Compulsive Scale (YBOCS) score of 25 or more; (ii) documented highly treatment refractory $\mathrm{OCD}$, including at least three adequate trials of different serotonin reuptake inhibitors (e.g., fluoxetine, sertraline, fluvoxamine, paroxetine, citalopram, escitalopram, and clomipramine) for at least 3 months at the maximum tolerated dose; augmentation of one of the SSRIs with clomipramine, a neuroleptic, or clonazepam (each for at least 2 weeks); and adequate behavior therapy (as indicated by a cognitive behavior therapist with substantial expertize in OCD treatment); (iii) drug-free or on a stable drug regimen for at least 6 weeks before study entry. Patients' demographics, baseline treatment and optimal stimulation parameters are presented in Table 1. All six patients (four PD patients and two OCD patients) are included in the intra-operative and post-operative analysis (Fig. 2). The second OCD patient declined post-operative follow-up after seven recording sessions. Therefore, only five patients (four PD patients and one OCD patient) are included in the analysis presented in Figs. 3 and 4. This study was authorized and supervised by the IRB of Hadassah Medical Center (no. 0403-13-HMO) and the Israel Ministry of Health (no. HT6752). Clinical Trials Registration number: NCT01962194

\section{Surgery planning and electrophysiological navigation}

The deep brain target chosen in this study for OCD patients was the assumed border between the associative and limbic areas of the $\mathrm{STN}^{17}$. This was $2 \mathrm{~mm}$ anterior to and $1 \mathrm{~mm}$ medial to the target that is used for PD patients ${ }^{17}$. Surgical technique has been previously described $^{24}$. Briefly, target was chosen before surgery according to high resolution T2 weighted MR images and trajectory planning was carried out according to $\mathrm{T} 1+$ Gadolinium MR images that were fused with high resolution CT acquired in the morning of surgery. During the surgery, the boundaries and the subdomains of the target nucleus were verified by microelectrode electrophysiological recordings of multi-unit spiking activity along the trajectory. Intra-operative analysis of the microelectrode spontaneous spiking activity recordings along the surgical trajectory, response of spiking activity to passive and active movements of the contralateral limbs and detecting the clinical effects of stimulation at the target assisted with the final localization of the permanent DBS electrode. Intra-operative recordings include multi-unit activity without spike sorting, so no analysis of discharge rate/pattern of single units can be made.

\section{Intra-operative electrophysiological recordings}

In each STN trajectory two electrodes, separated by $2 \mathrm{~mm}$ each, were advanced simultaneously in the vertical dimension. The typical distance between vertical recording sites before the entrance to the STN was $200-400 \mu \mathrm{m}$. The typical distance between recording sites within the STN was $100 \mu \mathrm{m}$. Areas with smaller number of recording sites were postoperatively interpolated to the general number of recording sites. Neurophysiological activity was recorded via polyamide coated tungsten microelectrodes (AlphaOmega Engineering, Nazareth, Israel) with impedance of around of $0.3-0.7 \mathrm{M} \Omega$ (measured at $1 \mathrm{KHz}$ ). The data were acquired with the NeuroOmega system (AlphaOmega Engineering, Nazareth, Israel). Analysis of intra-operative data had been previously described ${ }^{24}$. The signal was amplified by 20 , band-passed from 0.7 to $9000 \mathrm{~Hz}$ using a hardware four-pole Butterworth filter, and sampled at $44 \mathrm{kHz}$ by a 16-bit A/D converter (using $\pm 1.25 \mathrm{~V}$ input range). 


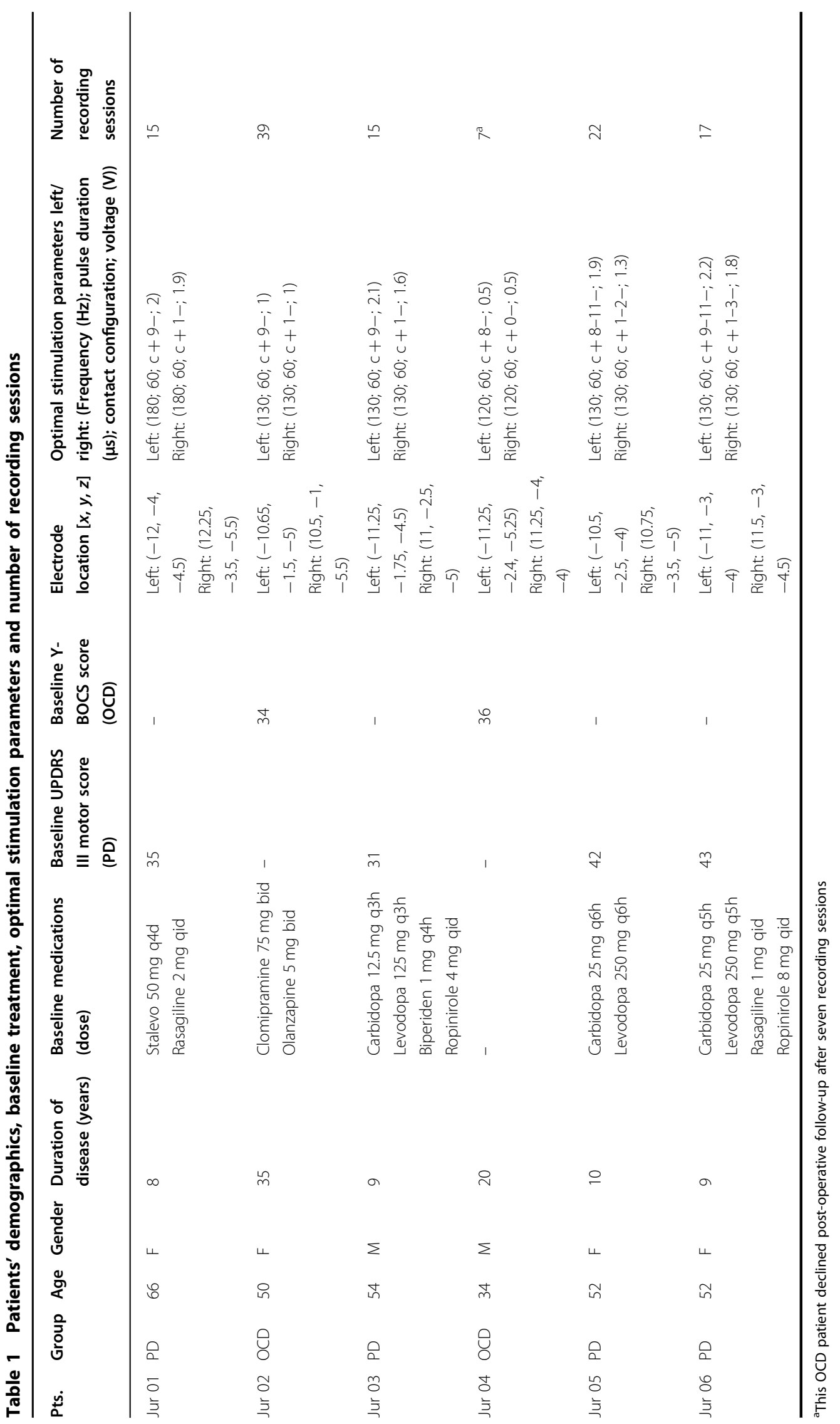




\section{Post-operative clinical assessment and electrophysiological recordings}

Electrophysiological post-operative LFPs (local field potentials) recordings were acquired in an outpatient setting. Patients came for clinical evaluation and recording sessions every 2-4 weeks for a period of 6-12 months. In every recording session, clinical assessments by a psychiatrist (RE) and neurologist (DA and EL) also took place. These included formal psychiatric assessments, including the Yale-Brown Obsessive-Compulsive Scale (Y-BOCS).

The implanted brain leads (model 3389; Medtronic, Inc., Minneapolis, MN) consisted of four contacts, each with a diameter of $1.27 \mathrm{~mm}$ and length of $1.5 \mathrm{~mm}$ spaced by $0.5 \mathrm{~mm}$ intervals. The lead was placed along the dorsoventral axis of the $\mathrm{STN}$, and contacts were numbered from 0 (ventral) to 3 (dorsal). We used bipolar macroelectrode recordings inside the STN. This method has been shown to overcome volume conductance effects and reflect locally generated neuronal activity ${ }^{24}$. The signal was amplified by 2000, band-passed from 0.5 to $100 \mathrm{~Hz}$, using a 3 pole Butterworth filter, and sampled at $422 \mathrm{~Hz}$ by a 10 -bit $A / D$ converter (using $\pm 2 \mathrm{~V}$ input range). The behavioral tasks and EEG data were synchronized with the LFP $(\mathrm{PC}+\mathrm{S})$ recordings using a short period $(5-10 \mathrm{~s})$ of low frequency stimulation pulse $(5 \mathrm{~Hz})$ at the beginning and end of each test.

Two types of recording were conducted: rest-state recordings and recordings during tasks.

\section{Rest-state recordings}

Participants were instructed to sit quietly for $3 \mathrm{~min}$ while bipolar recordings were obtained bilaterally for $30 \mathrm{~s}$ from each pair of electrodes $(0-1,0-2,0-3,1-2,1-3$, $2-3)$. Rest state recordings were obtained from OCD patients at the beginning and at the end of each session. Rest state recordings were obtained from PD patients at the beginning of each session while they were off medication (i.e., overnight cessation of all parkinsonian medications) and at the end of each session while they were on medication (i.e., at least an hour after taking their regular parkinsonian medications).

\section{Recordings during tasks}

Electrophysiological activity was recorded while subjects participated in four tasks:

The OCD provocative images task In this task patients were exposed to a series of individually tailored OCDrelevant stimuli, aversive stimuli and neutral stimuli. Images were taken from a validated set of pictures, Berlin OCD Picture Set (BOCD-PS), which was previously used by Simon et $\mathrm{al}^{25}$. As the ability to provoke OCD symptoms is culturally-dependent we validated a culture adjusted religious OCD provocation test in healthy Israeli volunteers. Prior to surgery, the OCD patients rated 250 pictures on a 0-10 scale according to the degree of their individual OCD arousal. A patient-specific OCD-relevant paradigm including neutral, aversive, or OCD provocative figures was built for each OCD patient. PD patients were presented with the paradigm of the OCD patient to whom they served as a control. During the recordings subjects were instructed to look at the screen in front of them. At every trial a figure appeared for 3 or $4 \mathrm{~s}$, followed by a black screen presented for an intertrial interval (ITI) of one second.

The doubt task This task was based on the hypothesis that a basic feature of OCD is patients' difficulty to deal with daily doubts. Subjects were instructed to look at a screen in front of them. Each trial started with a $2 \mathrm{~s}$ presentation of a predictive cue which could have been a plus sign, a minus sign, or a question mark, followed by $1.5 \mathrm{~s}$ presentation of an image from the Geneva Affective Picture Database (GAPED) ${ }^{26}$. Plus and minus signs predicted the type of the following image, neutral or aversive, respectively, with $100 \%$ certainty. A question mark was followed with either an aversive or a neutral Image with equal probability.

The auditory Go-NoGo task In this task a series of 120 tones, composed of frequent $(82 \%)$ high pitch $(1200 \mathrm{~Hz})$ tone and deviant $(18 \%)$ low pitch $(300 \mathrm{~Hz})$ tone were played in a pseudo-random order. The tone duration was $250 \mathrm{~ms}$ followed by $1000 \mathrm{~ms}$ ITI. Participants were instructed to press a handed button as fast as possible after the frequent "go" tone and to avoid pressing the button after the deviant "no go" tone. Subjects response was classified to four groups: "hit"-pressing the button in go trials, "miss"- not pressing the button in go trials, "commission error" $(\mathrm{CE})$ - pressing the button in no-go trials, and "correct rejection" (CR) - not pressing the button in no-go trials.

The emotional voice recognition task In this task subjects listened to and were asked to mentally recognize pseudo-randomized set of emotional voices from the Montreal affective voices (MAV) database, a validated database which consists of negative, neutral, and positive non-verbal male and female voices ${ }^{27}$.

In each recording session patients participated in all four tasks while bipolar recordings were obtained bilaterally for about 20-25 min from one pair of electrodes (either a ventral (0-2) or a dorsal (1-3) pair of electrodes). Task order was as follows: first, the OCD provocative images task; second, the doubt task; third, the Go-NoGo task; forth, the emotional voice recognition task. Each task lasted 3-5.5 min and the interval between tasks was 2-15 min. 


\section{Stimulation parameters and medications}

Monopolar review was conducted two weeks after surgery. Each contact was tested separately for the boundaries of the therapeutic window. Stimulation pulse duration was $60 \mu$ s with frequency and voltage adjusted to the individual patient. The therapeutic contact/s and configuration of contacts were selected according to the best immediate clinical effect together with the intraoperative data and anatomical localization of each contact. The OCD patients' medical treatment remained stable with small changes in benzodiazepine medication dosage. The PD patients' dopamine replacement treatment was reduced by $\sim 50-75 \%$ according to their clinical state and the recommendation in the literature.

\section{Data analysis}

\section{Artifacts removal}

In some of the recordings (mainly those containing the most ventro-medial contact) an electrocardiogram (ECG) artifact contaminated the neural activity signal. ECG pulses were identified by their high peak (above $1.2 \mathrm{SD}$ over the mean) and regularity $(\mathrm{CV}<0.22)$. In order to clean the data we adopted a template subtraction approach. We averaged all occurrences of the ECG signal in each recording to create a template, and then subtracted that template from every occurrence of the ECG artifact using linear regression to achieve optimal fit with the data. The efficiency of this method was evaluated by calculating the area under the spectrum and demanding its value to be below a visually set threshold. The presented data was recorded while stimulation was turned off, so no stimulation artifacts were recorded.

\section{Spectral analysis}

Data was band-pass filtered between 0.5 and $100 \mathrm{~Hz}$ using 4 poles Butterworth filter. Rest PSD was calculated using the Welch method with hamming window of $1 \mathrm{~s}$ and $50 \%$ overlap, and frequency resolution of $0.5 \mathrm{~Hz}$. $50 \mathrm{~Hz}$ noise was extracted from the PSD and replaced with estimated values using Piecewise Cubic Hermite interpolation (PCHIP). Spectrum was than normalized by dividing it by its total power. For presentation purposes we flatten the spectrum by $1 / \mathrm{f}$ normalization $\left(\mathrm{P}^{\prime}(\mathrm{f})=\mathrm{P}(\mathrm{f})\right.$ " $f$; ( $\mathrm{P}^{\prime}, \mathrm{P}$-original, normalized power, $\mathrm{f}$-frequency). In order to compare STN rest spectral activity in OCD, PD on medication and PD off medication groups we calculated for each patient the PSD of the first five rest recording sessions starting a week after the surgery. We performed on these PSDs a one way ANOVA test for every frequency in the range of $3-80 \mathrm{~Hz}$ with $0.5 \mathrm{~Hz}$ resolution, and used Bonferroni correction to control for multiple comparisons. According to the results of this test we chose our frequencies of interest for further analysis to be theta: $6.5-8 \mathrm{~Hz}$ and high beta: $25-35 \mathrm{~Hz}$.

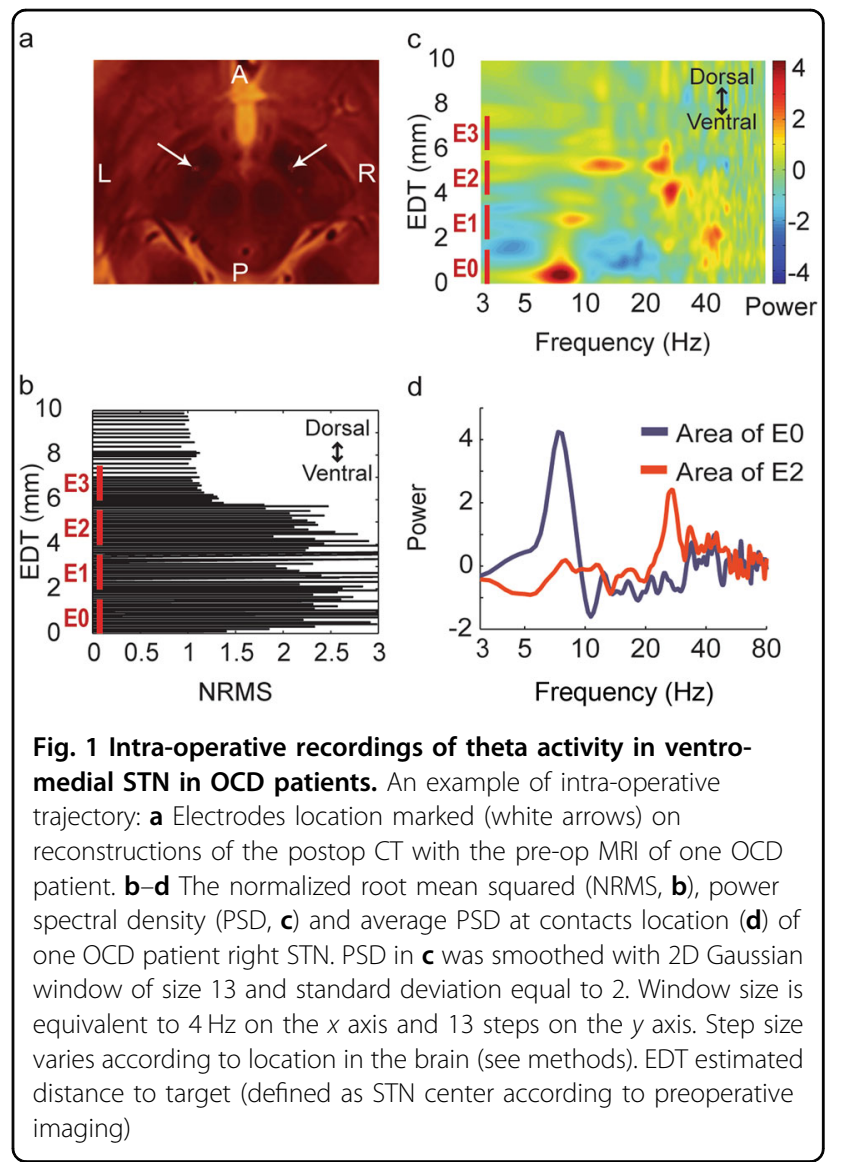

\section{Y-BOCS and theta/high beta correlations}

One of our OCD patients, Jur02, participated in the study for over a year, during which her clinical state varied substantially. That allowed us to test for possible correlation between OCD clinical state and STN neuronal activity changes within the same patient. Based on the results of spectral activity in this patient (Fig. 1) we decided to focus on theta $(6.5-8 \mathrm{~Hz})$ and high beta $(25-35 \mathrm{~Hz})$ power recorded from the left and right ventral (E0-E2 and E8-E10 contacts) and dorsal (E1-E3 and E9-E11 contacts) STN. Power at a specific frequency range was defined as the difference between the peak value at that band and the closest minima. If there was no local maximum point at the frequency band the power value was set to zero. In days with more than one rest recording sessions, we averaged results of all sessions so that every day contributed one data point to the correlation analysis. Finally, a Spearman's rank-order correlation was run to determine the relationship between clinical state and ventral STN theta and high beta power.

\section{Tasks analysis}

Data from right and left ventral (E0-E2 and E8-E10 contacts) and dorsal (E1-E3 and E9-E11 contacts) recording sites were included in the analysis. Single trials 
were extracted, and time frequency analysis was carried out using complex Morlet wavelet transform with a wavelet constant of 12 and center frequency bins ranging from 6.5 to $8 \mathrm{~Hz}$. The result scalograms were corrected by subtraction of the average power at rest for each frequency and averaged above frequencies. Statistical analysis was carried out on averaged theta activity during time periods that were determined according to manual detection of response to task events. In the go-no go task statistical analysis was carried out on averaged theta activity during 0.4-1 s after the cue. In OCD provocative images task, the time-point for statistical analysis was $1-1.5 \mathrm{~s}$ after image presentation. In the doubt task the time-point for statistical analysis was $0.5-2 \mathrm{~s}$ after cue presentation. In emotional voices task the time-point for analysis was $0-1 \mathrm{~s}$ after voice onset. Noisy traces were identified visually and excluded from further analysis and outliers with $\mathrm{z}$ score higher than 2 were excluded. We conducted mixed ANOVA test with two factors: group (3: OCD, PD on medication, PD off medication) X condition (repeated measure factor, 3: task conditions). In case of a significant effect we conducted simple main effects procedure with Bonferroni correction for multiple comparisons.

\section{Statistics}

A threshold of 0.05 was used to establish statistical significance. All statistical tests were two-tailed. Data analyses were performed using Matlab 2013b (Mathworks, MA, USA) and statistical analyses were performed using SPSS 24.0 (IBM SPSS Statistics for Windows. Armonk, NY: IBM Corp).

\section{Results}

Intraoperative microelectrode spiking activity recordings revealed distinct theta-oscillatory activity in the ventro-medial STN of OCD patients (Fig. 1a-d). Betaoscillatory activity was identified in the dorso-lateral region of the STN in Parkinson's patients and most interestingly also in OCD patients (Fig. 1b-d). Multiple macroelectrode LFP recordings during the first postoperative year clearly demonstrated that OCD patients had beta-oscillatory activity mainly at the dorso-lateral STN and theta-oscillatory activity mainly at the ventromedial STN (Fig. 2a, b). Comparison of spectral activity from dorsal and ventral STN revealed significant differences in the theta and beta band frequencies between OCD and PD (on and off medication) patients (Fig. 2c, d).

The first OCD patient's clinical state was closely monitored and rated with the Yale-Brown Obsessive-Compulsive Scale (Y-BOCS) for the first postoperative year (Fig. 3a). As the OCD symptoms decreased (lower Y-BOCS score) the theta activity at the ventro-medial STN increased (Fig. 3b, c). After 8 months of treatment we observed an unexplained increase in the patient's OCD symptoms severity
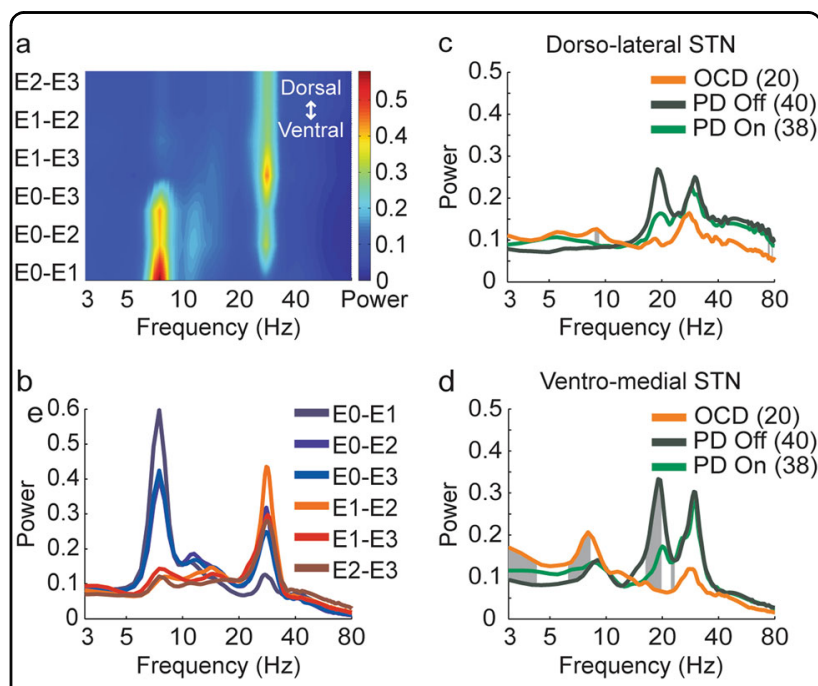

Fig. 2 Post-operative recordings of beta and theta activity in dorso-lateral and ventro-medial STN in OCD and PD patients. $a, b$ Average PSD ( $N=52$ ) of post-operative recordings of one OCD patient (Same patient as in Fig. 1), arranged spatially (a) and in a spectrogram (b). c, d Average spectral activity from dorsal (c; bipolar pairs: E2-E3 and E10-E11) and ventral (d; bipolar pairs: E0-E2 and E8-E10) STN of OCD and PD (on and off medication) patients in five recording sessions from one week to three months post operation. Gray areas indicate frequencies in which the difference between the groups is significant $(p<0.05$ after Bonferroni correction)

that was accompanied by a decline in theta power. After another few weeks as the OCD symptoms decreased again, the theta activity increased. The severity of OCD symptoms was inversely correlated with the theta activity at the ventro-medial right $\left(r_{\mathrm{s}}=-0.65, p=0.01\right)$ and left $\left(r_{\mathrm{s}}=\right.$ $-0.62, p=0.02)$ STN, but not with high beta activity in those areas (Fig. 3c, d). The severity of OCD symptoms was also inversely correlated with high beta activity in the dorsolateral left STN $\left(r_{\mathrm{s}}=-0.6, p=0.03\right)$, but not right $\left(r_{\mathrm{s}}=\right.$ $-0.28, p=1$ ) dorso-medial STN (Fig. 3e-g). Theta activity in the dorso-medial STN did not correlate with Y-BOCS scores (Fig. 3f).

Behavioral tasks, including visual OCD provocation and doubt tests, auditory cognitive Go-NoGo paradigm and emotional voices recognition test downregulated ventromedial STN theta activity in OCD patients but not in PD patients (Fig. 4a). OCD patients responded to OCD provocative images, commission errors in the Go-NoGo test and positive emotional voices in a decrease in theta activity. However, no OCD-specific responses in thetaband activity were found in doubt test and in response to emotional voices.

\section{OCD provocative task (Fig. $4 \mathrm{~b}, \mathrm{c}$ upper row)}

Significant group $\times$ image interaction $(F(4,58)=3.653$, $p=.010)$, as well as significant main effect of images ( $\mathrm{F}$ $(2,58)=6.456, p=.003)$ were found in the visual $\mathrm{OCD}$ 


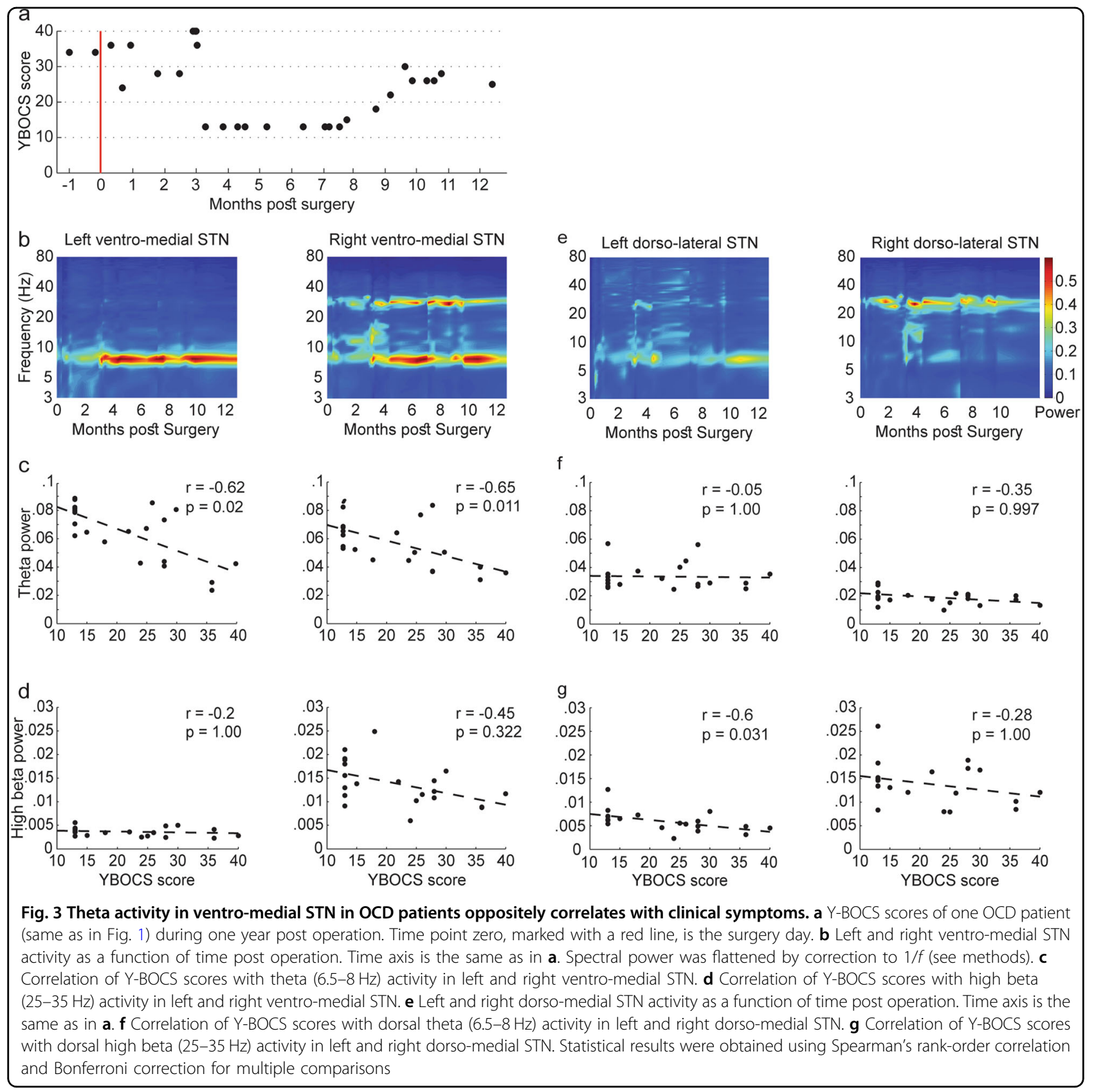

provocation test. Post hoc test revealed that OCD patients responded to OCD provocative images in a decrease in theta activity $(-.054 \pm .014)$ and that response was more robust than their response to neutral $(-010 \pm .013, p$ $=.0001)$ and aversive $(-018 \pm .015, p=.011)$ images. In addition a significant difference between PD patients on medication response to neutral $(.027 \pm .018)$ and aversive $(-.007 \pm .016, p=.045)$ images was found.

\section{Doubt task (Fig. 4b, c second row)}

A main effect of cue type $(\mathrm{F}(2,50)=3.464, p=.039)$ with no interaction effect was found in the visual doubt test. Post hoc tests showed that across all groups, activity after an ambiguous cue $(.011 \pm .006)$ was higher than after positive cue $(-.006 \pm .008, p=.029)$.

\section{Go-NoGo task (Fig. 4b, c third row)}

A significant group $\times$ trial interaction $(\mathrm{F}(4,76)=6.943$, $p=.00008)$ was found in the Go-NoGo paradigm. Post hoc tests showed that in OCD patients response to tone in commission error trials $(-021 \pm .016)$ was lower than in hit $(.061 \pm .01, p=.0001)$ and correct rejection trials $(.04$ $\pm .018, p=.013)$. OCD response to tone in hit trial was significantly higher than that of PD off medication (.007 


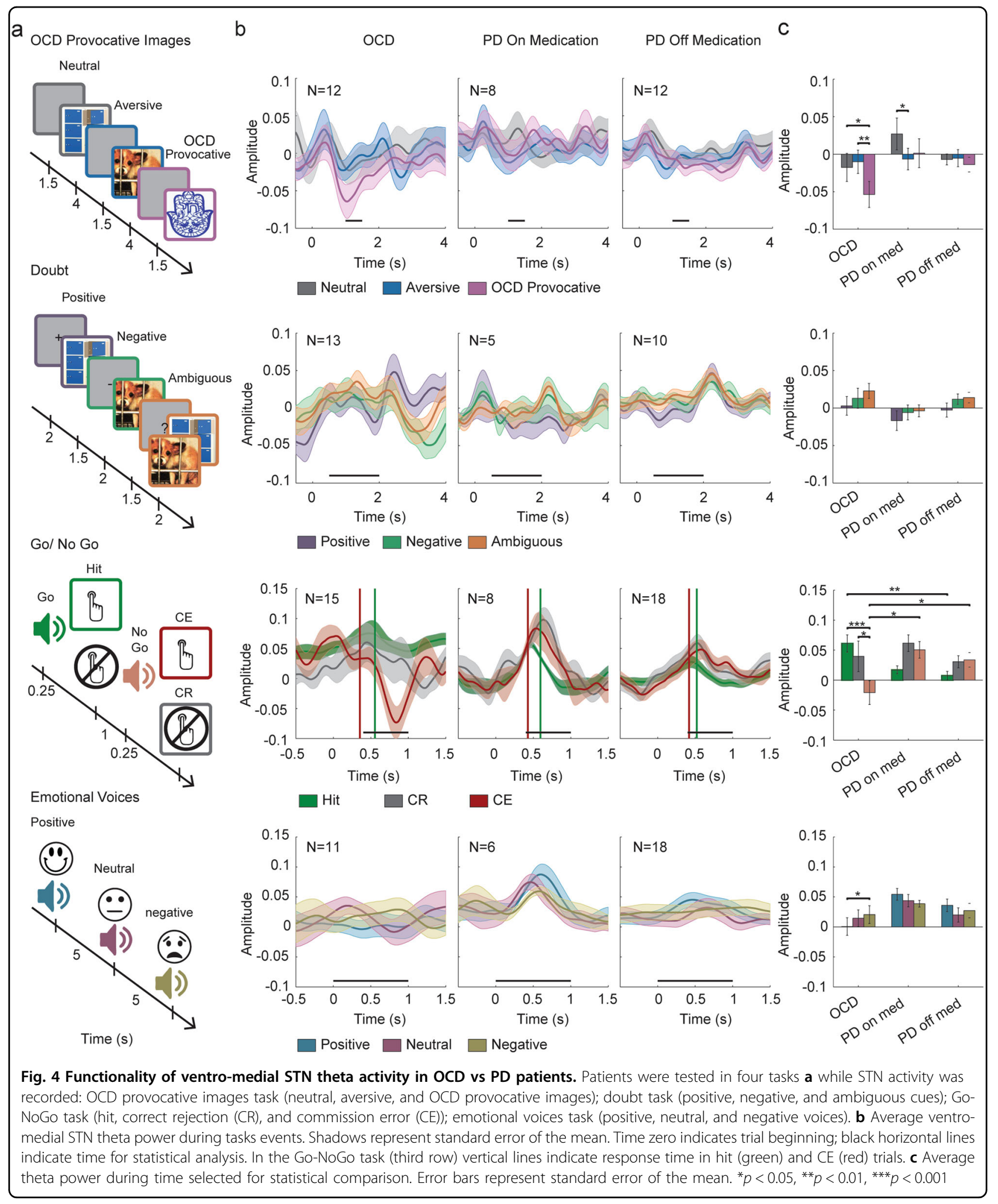


$\pm .01, p=.002$ ), and OCD response to tone in commission error trials was negative and opposite to PD response on $(.051 \pm .021, p=.031)$ and off medication $(.034 \pm .014, p$ $=.041)$. Vertical lines indicate response time in hit (green) and CE (red) trials.

\section{Emotional voices task (Fig. 4b, c forth row)}

A significant group $\mathrm{x}$ voice interaction $(\mathrm{F}(4,64)=2.858$, $p=.030$ ) was found in the emotional voices recognition test. Post hoc tests showed that OCD patients' theta activity after positive voices $(.001 \pm .013)$ was significantly lower than after negative cue $(.021 \pm .014, p=.026)$. No significant difference was found in PD patients' responses to the voices.

No significant interaction effects of patient condition (OCD or PD) and task events were found in dorsal activity in the OCD provocation test, the doubt test and the emotional voices recognition test (Supplementary Fig. 1). In the Go-NoGo paradigm a main effect of trial type $(\mathrm{F}(2,84)=$ 10.533, $p=.00008$ ) was found, with no interaction with group factor (Supplementary Fig. 1a-b third row).

\section{Discussion}

Our results are based on two methodological advantages. First, we used the investigational device, Activa PC $+\mathrm{S}$ (Medtronic Inc.), that enables long-term LFPs recording and a correlation between STN activity and patient's clinical state. Second, although OCD patients cannot be compared to healthy controls we used a matching control group of PD patients. In this study we have found a subcortical mechanism for OCD. First, the dorsal (motor) area of the STN in OCD patients displays a beta $(25-35 \mathrm{~Hz})$ oscillatory activity similar to PD patients whereas the ventral (limbic-cognitive) area of the STN displays distinct theta $(6.5-8 \mathrm{~Hz})$ oscillatory activity only in OCD patients. Second, these STN theta oscillations decrease with provocation of OCD symptoms and are inversely correlated with symptoms severity over time.

Our observations of high beta $(25-35 \mathrm{~Hz})$ oscillatory activity at dorso-lateral (motor) STN in OCD and PD patients challenge the concept that beta oscillations are pathognomonic for akinetic movement disorders. Beta oscillations have been suggested as a biomarker to trigger closed loop DBS for $\mathrm{PD}^{28-30}$. These results suggest a reconsideration of the optimal characteristics of these oscillations (in the temporal and/or frequency domains) for adaptive DBS paradigms ${ }^{31}$.

Another major finding is the unique theta oscillations recorded in the ventro-medial STN in OCD patients, correlated with the severity of OCD symptoms and modulated by provocation of OCD symptoms and other emotional and cognitive tasks. Ventro-medial STN theta activity might represent the level of inhibition of OCD symptoms: theta activity increases with successful inhibition of $\mathrm{OCD}$ symptoms and decreases with the failure to inhibit OCD symptoms. Previous intraoperative recordings of STN neurons in OCD patients have demonstrated a low firing rate and increased delta $(1-4 \mathrm{~Hz})$ activity but have not shown a correlation between OCD severity and mean discharge rate ${ }^{19,20}$. OCD symptoms were related to changes in different frequency bands, with no clear preferential oscillatory pattern in postoperative recordings from two patients during unexpected provocation of OCD symptoms that occurred during a cognitive task ${ }^{22}$. STN activity during a decision-making task revealed that checking behavior was associated with a higher STN firing rate and that stopping decisions were associated with beta band activity ${ }^{22,32}$. Recordings from other DBS targets in OCD patients revealed high frequency discharge and variability of interspike intervals in the caudate nucleus $(n=2)$ and lack of alpha-band $(8-14 \mathrm{~Hz})$ oscillatory activity in the bed nucleus of stria terminalis (in 5 OCD patients vs. 7 major depression patients) ${ }^{33,34}$. In a recent study using combined LFP and magnetoencephalography (MEG) recordings, thetacoupling was evident between the STN and the anterior cingulate cortex ${ }^{23}$.

The differences in investigational methods might explain the variability in oscillatory activity between this study and the previous literature. All previous electrophysiological recordings in OCD patients were performed either intra-operatively or in the first post-operative days. Awake DBS surgery involves considerable amount of stress and anxiety that might alter neuronal activity. The short post-operative period might be confounded with micro-lesion, local edema and insertional effect that impact the electrophysiological activity. Our results obtained in a neutral outpatient set-up over a long period of clinical follow-up emphasize the importance of theta oscillation in the physiology of OCD symptoms. Nevertheless, our study is limited by a small number of OCD patients and further verification of these results in a larger sample of patients is needed.

Few studies have correlated STN low frequency oscillations (theta $(4-8 \mathrm{~Hz})$ and alpha $(8-13 \mathrm{~Hz})$ frequency bands) with motor, cognitive, and emotional clinical effects. STN low frequency $(4-10 \mathrm{~Hz})$ oscillations were correlated with levodopa-included dyskinesia and impulse control disorders in PD patients ${ }^{35,36}$. Presentation of emotional stimuli has been shown to reduce STN alpha oscillatory activity in PD patients ${ }^{37-39}$. High-conflict tasks has been shown to increase STN theta oscillatory activity $^{40-43}$ and conflictual moral stimuli have been shown to increase STN theta and alpha $(5-13 \mathrm{~Hz})$ oscillatory activity $^{44}$. Slowing the responses in a simple responseinhibition task has been correlated with increased STN alpha oscillatory activity ${ }^{45}$.

Our results are in line with these previous reports, showing a correlation between STN theta oscillatory 
activity and response inhibition. We show an increased theta oscillatory activity during response and responseinhibition in the Go-NoGo task both in PD and OCD patients (Fig. 4 third row). However, failure to inhibit responses (commission errors) is correlated with a decreased theta oscillatory activity in OCD patients. Similarly, provocation of OC symptoms (OCD provocation task, Fig. 4 first row) is correlated with a decreased theta oscillatory activity in OCD patients. Both commission errors and $\mathrm{OC}$ symptoms are a failure to inhibit the urge to inappropriate thoughts or behaviors and are correlated with decreased STN theta activity. Long-term successful inhibition of the urge to inappropriate thoughts or behaviors is clinically represented by the decreased severity of OCD symptoms (decreased Y-BOCS score) and is correlated with increased theta oscillatory activity (Fig. 3).

It is worth noting that comparing OCD and PD patients has some limitation related to the different diagnosis, age, duration of disease, comorbid illness, medication intake, and so on. Another possible difference between the OCD and PD patients is the site of electrode implantation. In OCD patients the electrodes were $2 \mathrm{~mm}$ anterior to and $1 \mathrm{~mm}$ medial to the target that was used for PD patients. Analysis of the intra-operative recordings of STN in both OCD and PD patients enabled identification of the beta oscillatory activity and differentiation of the dorso-lateral area from the ventro-medial area of the STN for each patient (see an example in Fig. 1b-d).

In conclusion, we show that STN theta oscillatory activity can modulate cognitive and emotional aspects of OCD. We suggest that theta activity (or decreased theta activity) at the ventro-medial STN might be used as a biomarker for closed-loop feedback-controlled stimulation to treat OCD: specific stimulation that enhances theta activity might reduce the severity of OCD symptoms.

\section{Acknowledgements}

The authors acknowledge Scott Stanslaski and Tim Denison (Medtronic, Inc) contribution to the development and clinical implantation of the Activa PC+ $S$ device and thank them for a series of helpful recommendations about longterm LFP recordings.

\footnotetext{
Author details

'Department of Medical Neurobiology (Physiology), Institute of Medical Research - Israel-Canada, the Hebrew University-Hadassah Medical School, Jerusalem, Israel. ${ }^{2}$ The Edmond and Lily Safra Center for Brain Research, the Hebrew University, Jerusalem, Israel. ${ }^{3}$ The Brain Division, Hadassah-Hebrew University Medical Center, Jerusalem, Israel. ${ }^{4}$ Grenoble Institute of Neuroscience, Grenoble, France. ${ }^{5}$ The Center for Functional and Restorative Neurosurgery, Hadassah-Hebrew University Medical Center, Jerusalem, Israel. ${ }^{6}$ Department of Neurosurgery, University of California San Francisco, San Francisco, CA, USA. ${ }^{7}$ School of Social Work, Bar Ilan University, Ramat Gan, Israel. ${ }^{8}$ Department of Psychiatry, Functional Neuroimaging Laboratory, Brigham and Women's Hospital, Harvard Medical School, Boston, MA, USA
}

\section{Author contributions}

P.R., O.M., A.S.B., D.A., E.L., A.C., I.T., S.A.F., T.M., M.G., H.B., Z.I., and R.E. were engaged in study conception and interpretation and contributed to the critical revision of the manuscript. M.O., A.S.B., A.C., I.T., H.B., Z.I., and R.E. were involved in intra-operative and post-operative recordings. D.A., E.L., A.C., S.F., H.B., Z.I., and R.E. were involved in clinical neurology and psychiatry follow-up. O.M., A.S.B., S.A.F., T.M., M.G., and R.E. developed the OCD behavioral tasks. P.R. O.M., A.S.B., D.A., H.B., Z.I, and R.E. analyzed the electrophysiological data. P.R., O.M., H.B., Z.I., and R.E. prepared the manuscript. All authors discussed the results, assisted in the preparation of the manuscript and approved the final version.

\section{Conflict of interest}

The Activa PC $+S$ devices were generously provided by Medtronic Inc, MA, USA. The study was partially supported by grants from the NARSAD Young Investigator Grant (R.E.), the Israel Science Foundation-ISF no. 1129/12 (R.E. and H.B.), the Israel-US Binational Science Foundation-BSF no. 2011410 (R.E. and H. B.), the Magnet program of the Office of the Chief Scientist (OCS) of the Ministry of Economy of Israel (H.B.) and the Adelis foundation grant (Z.I. and $\mathrm{H}$. B.). The remaining authors declare no competing interests.

\section{Publisher's note}

Springer Nature remains neutral with regard to jurisdictional claims in published maps and institutional affiliations.

Supplementary Information accompanies this paper at https://doi.org/ 10.1038/s41398-018-0165-z.

Received: 27 February 2018 Accepted: 22 April 2018

Published online: 18 June 2018

\section{References}

1. Haber, S. N. \& Heilbronner, S. R. Translational research in OCD: circuitry and mechanisms. Neuropsychopharmacology 38, 252-253 (2013).

2. Ahmari, S. E. \& Dougherty, D. D. Dissecting OCD circuits: from animal models to targeted treatments. Depress Anxiety 32, 550-562 (2015).

3. Bourne, S. K., Eckhardt, C. A., Sheth, S. A. \& Eskandar, E. N. Mechanisms of deep brain stimulation for obsessive compulsive disorder: effects upon cells and circuits. Front Integr. Neurosci. 6, 29 (2012).

4. Lipsman, N., Giacobbe, P. \& Lozano, A. M. Deep brain stimulation in obsessivecompulsive disorder: neurocircuitry and clinical experience. Handb. Clin. Neurol. 116, 245-250 (2013).

5. Lapidus, K. A., Stern, E. R., Berlin, H. A. \& Goodman, W. K. Neuromodulation for obsessive-compulsive disorder. Neurotherapeutics 11, 485-495 (2014).

6. Mulders, A. E. P. et al. Deep brain stimulation of the subthalamic nucleus in obsessive-compulsive disorder: neuroanatomical and pathophysiological considerations. Eur. Neuropsychopharmacol. 26, 1909-1919 (2016).

7. Mallet, L. et al. Subthalamic nucleus stimulation in severe obsessivecompulsive disorder. N. Engl. J. Med 359, 2121-2134 (2008). STOC Study Group.

8. Le Jeune, F. et al. French stimulation dans le trouble obsessionnel compulsif (STOC) study group. Decrease of prefrontal metabolism after subthalamic stimulation in obsessive-compulsive disorder: a positron emission tomography study. Biol. Psychiatry 68, 1016-1022 (2010).

9. Chabardès, S. et al. Deep brain stimulation for obsessive-compulsive disorder: subthalamic nucleus target. World Neurosurg. 80, S31.e1-e8 (2013).

10. Blomstedt, P., Sjöberg, R. L., Hansson, M., Bodlund, O. \& Hariz, M. I. Deep brain stimulation in the treatment of obsessive-compulsive disorder. World Neurosurg. 80, e245-e253 (2013).

11. Hamani, C. et al. American Society for Stereotactic and Functional Neurosurgery; Congress of Neurological Surgeons; CNS and American Association of Neurological Surgeons. Deep brain stimulation for obsessive-compulsive disorder: systematic review and evidence-based guideline sponsored by the American Society for Stereotactic and Functional Neurosurgery and the Congress of Neurological Surgeons (CNS) and endorsed by the CNS and American Association of Neurological Surgeons. Neurosurgery 75, 327-333 (2014). 
12. Kohl, S. et al. Deep brain stimulation for treatment-refractory obsessive compulsive disorder: a systematic review. BMC Psychiatry 14, 214 (2014).

13. Morishita, T. et al. Surgical neuroanatomy and programming in deep brain stimulation for obsessive compulsive disorder. Neuromodulation 17, 312-319 (2014).

14. Fries, P. A mechanism for cognitive dynamics: neuronal communication through neuronal coherence. Trends Cogn. Sci. 9, 474-480 (2005).

15. Brown, P. \& Williams, D. Basal ganglia local field potential activity: character and functional significance in the human. Clin. Neurophysiol. 116, 2510-2519 (2005).

16. Kühn, A. A., Kupsch, A., Schneider, G. H. \& Brown, P. Reduction in subthalamic 8-35 Hz oscillatory activity correlates with clinical improvement in Parkinson's disease. Eur. J. Neurosci. 23, 1956-1960 (2006).

17. Moran, A., Bergman, H., Israel, Z. \& Bar-Gad, I. Subthalamic nucleus functional organization revealed by parkinsonian neuronal oscillations and synchrony. Brain 131, 3395-3409 (2008).

18. de Hemptinne, C. et al. Therapeutic deep brain stimulation reduces cortical phase-amplitude coupling in Parkinson's disease. Nat. Neurosci. 18, 779-786 (2015).

19. Welter, M. L. et al. French Stimulation dans Trouble Obsessionnel Compulsif (STOC) Study Group. Basal ganglia dysfunction in OCD: subthalamic neuronal activity correlates with symptoms severity and predicts high-frequency stimulation efficacy. Transl. Psychiatry 1, e5 (2011).

20. Piallat, B. et al. Subthalamic neuronal firing in obsessive-compulsive disorder and Parkinson disease. Ann. Neurol. 69, 793-802 (2011).

21. Bastin, J. et al. Changes of oscillatory activity in the subthalamic nucleus during obsessive-compulsive disorder symptoms: two case reports. Cortex $\mathbf{6 0}$, 145-150 (2014).

22. Burbaud, P. et al. Neuronal activity correlated with checking behaviour in the subthalamic nucleus of patients with obsessive-compulsive disorder. Brain 136, 304-317 (2013). French Stimulation dans le Trouble Obsessionnel Compulsif Study Group.

23. Wojtecki, L. et al. Oscillatory coupling of the subthalamic nucleus in obsessive compulsive disorder [letter]. Brain 140, e56 (2017).

24. Marmor, O. et al. Local vs. volume conductance activity of field potentials in the human subthalamic nucleus. J. Neurophysiol. 117, 2140-2151 (2017).

25. Simon, D., Kischkel, E., Spielberg, R. \& Kathmann, N. A pilot study on the validity of using pictures and videos for individualized symptom provocation in obsessive-compulsive disorder. Psychiatry Res. 198, 81-88 (2012).

26. Dan-Glauser, E. S. \& Scherer, K. R. The Geneva affective picture database (GAPED): a new 730-picture database focusing on valence and normative significance. Behav. Res. Methods 43, 468-477 (2011).

27. Eitan, R. et al. Asymmetric right/left encoding of emotions in the human subthalamic nucleus. Front. Syst. Neurosci. 7, 69 (2013).
28. Rosin, B. et al. Closed-loop deep brain stimulation is superior in ameliorating parkinsonism. Neuron 72, 370-384 (2011)

29. Little, S. et al. Adaptive deep brain stimulation in advanced Parkinson disease Ann. Neurol. 74, 449-457 (2013).

30. Buot, A. C. et al. Adaptive deep brain stimulation for movement disorders: the long road to clinical therapy. Mov. Disord. 32, 810-819 (2017).

31. Accolla, E. A., Horn, A., Herrojo-Ruiz, M., Neumann, W. J. \& Kühn, A. A. Reply: oscillatory coupling of the subthalamic nucleus in obsessive compulsive disorder [letter]. Brain 140, e57 (2017).

32. Bastin, J. et al. Inhibitory control and error monitoring by human subthalamic neurons. Transl. Psychiatry 4, e439 (2014).

33. Guehl, D. et al. Neuronal correlates of obsessions in the caudate nucleus. Biol. Psychiatry 63, 557-562 (2008).

34. Neumann, W. J. et al. Different patterns of local field potentials from limbic DBS targets in patients with major depressive and obsessive compulsive disorder. Mol. Psychiatry 19, 1186-1192 (2014).

35. Alonso-Frech, F. et al. Slow oscillatory activity and levodopa-induced dyskinesias in Parkinson's disease. Brain 129, 1748-1757 (2006).

36. Rodriguez-Oroz, M. C. et al. Involvement of the subthalamic nucleus in impulse control disorders associated with Parkinson's disease. Brain 134 36-49 (2011).

37. Kühn, A. A. et al. Activation of the subthalamic region during emotional processing in Parkinson disease. Neurology 65, 707-713 (2005).

38. Brücke, C. et al. The subthalamic region is activated during valence-related emotional processing in patients with Parkinson's disease. Eur. J. Neurosci. 26, 767-774 (2007).

39. Huebl, J. et al. Oscillatory subthalamic nucleus activity is modulated by dopamine during emotional processing in Parkinson's disease. Cortex 60, 69-81 (2014).

40. Brittain, J. S. et al. A role for the subthalamic nucleus in response inhibition during conflict. J. Neurosci. 32, 13396-13401 (2012).

41. Zavala, B. et al. Subthalamic nucleus local field potential activity during the Eriksen flanker task reveals a novel role for theta phase during conflict monitoring. J. Neurosci. 33, 14758-14766 (2013).

42. Herz, D. M., Zavala, B. A., Bogacz, R. \& Brown, P. Neural correlates of decision thresholds in the human subthalamic nucleus. Curr. Biol. 26, 916-920 (2016).

43. Zavala, B. et al. Human subthalamic nucleus theta and beta oscillations entrain neuronal firing during sensorimotor conflict. Cereb. Cortex 27, 496-508 (2017).

44. Fumagalli, M. et al. Conflict-dependent dynamic of subthalamic nucleus oscillations during moral decisions. Soc. Neurosci. 6, 243-256 (2011).

45. Ghahremani, A. et al. Stopping and slowing manual and spoken responses: similar oscillatory signatures recorded from the subthalamic nucleus. Brain Lang. 176, 1-10 (2017). 